\title{
Strontium Ranelate Reduces Duration of Consolidation Phase in Distraction Osteogenesis: 2 Case Reports
}

\author{
Kishan Rao Subramaniam ${ }^{1}$, Ed Simor Khan ${ }^{1}$, Ahmad Hafiz Zulkifly ${ }^{1}$ and Ashwini Sood ${ }^{2}$ \\ ${ }^{1}$ Department of Orthopedic, International Islamic University Malaysia, Malaysia \\ ${ }^{2}$ Department of Orthopedic, Hospital Kulim Kedah, Malaysia \\ *Corresponding author: Kishan Rao Subramaniam, Department of Orthopedic, Malaysia
}

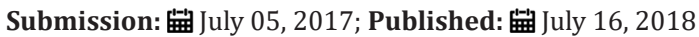

\begin{abstract}
Distraction osteogenesis a debilitating and time consuming procedure in orthopedics. The common indications for distraction osteogenesis are open fractures, non union and bone resection due to chronic osteomyelitis or large bone loss at initial time of trauma. It is often a limb salvage procedure but also has a role in cosmetic lengthening in short statures. In distraction osteogenesis, there are 3 main phases; latent phase, distraction phase and consolidation phase. Often, patient has to be on external fixator (eg: ilizarov, LRS) for the entire duration of the distraction osteogenesis until clinical union is achieved (frame time). Prolonged external fixation is uncomfortable for the patient and is associated with many complications. Strontium ranelate is widely used in treatment of osteoporosis and to prevent fractures in elderly population. It has dual action and it promotes osteoblastic activity and inhibits osteoclastic activities in bone cells. From our cases, we have found that strontium ranelate given to patients at the end of distraction phase of distraction osteogenesis have significantly reduced the duration of consolidation phase, thus shortening the duration of the patients on external fixator (frame time). Strontium ranelate is a cost effective and non invasive alternative and can be used to reduce patients' suffering, improve patients' compliance and reduce the disability period. However, further evaluation of the safety profile of the drug needs to be taken into consideration.
\end{abstract}

Keywords: Distraction osteogenesis; Bone transport; Frame time; Strontium ranelate; Consolidation phase

\section{Introduction}

Distraction osteogenesis was invented by Gavriil Abramovich Ilizarov from Russia in 1951 [1]. Distraction osteogenesisis a debilitating and time consuming procedure in orthopedics. The common indications for distraction osteogenesis are open fractures, non-union, bone resection due to chronic osteomyelitis or large bone loss at initial time of trauma [1-8]. It is often a limb salvage procedure but also has a role in cosmetic lengthening in short statures [3,9].Other known methods to treat large bone deficiencies are bone grafting (autograft, allograft or synthetic), membrane induction technique or also known as Masquelet technique, cortical strut grafting, or vascularised bone grafting [1,3,8-10]. Some literatures also have mentioned acute shortening of the fracture ends and bone lengthening later [7]. In bone transport, there are 3 main phases; latent phase, distraction phase and consolidation phase $[3,9]$. Patient has to be on external fixator (eg: ilizarov, LRS) for the entire duration of the bone transport until clinical union is achieved. Prolonged external fixation is uncomfortable for the patient and is associated with many complications such as pin site infections, pin dislodgement, mal-union, mechanical failure and poor cosmetic appearance $[1,8,9]$. It is the main reason for lack of compliance from patients, which is a significant setback for this otherwise a revolutionary procedure in orthopedics.

Strontium ranelate is used in treatment of osteoporosis. It has dual action; stimulates osteoblastic activity and inhibits osteoclastic activity [9,11-15]. It is widely used in treatment of osteoporosis and to prevent fractures in elderly population. From our cases, we have found that strontium ranelate given to patients at the end of distraction phase have significantly reduced the duration of consolidation phase of the regenerated part in these patients. Thus, reducing the duration the patients have to be on external fixator (frame time).

\section{Case Report}

\section{Patient 1}

19 years old boy sustained open fracture grade 3A of right distal $3^{\text {rd }}$ femur, done wound debridement+interlocking nailing of femur. Unfortunately, 5 months later patient presented with chronic osteomyelitis. $8.5 \mathrm{~cm}$ of bone was removed. Later, bone transport was started using LRS system. Bone transport was completed 3 months (87 days) later and bone grafting+plating was done at 
docking site. Patient was thereafter started on Strontium Ranelate $2 \mathrm{~g} / \mathrm{od}$. Below are the $\mathrm{x}$-rays of the patient. It shows that the new bone formed has consolidated well and docking site has united
LRS was removed earlier than the expected duration and patient is ambulating FWBA with single crutches (Figure 1).

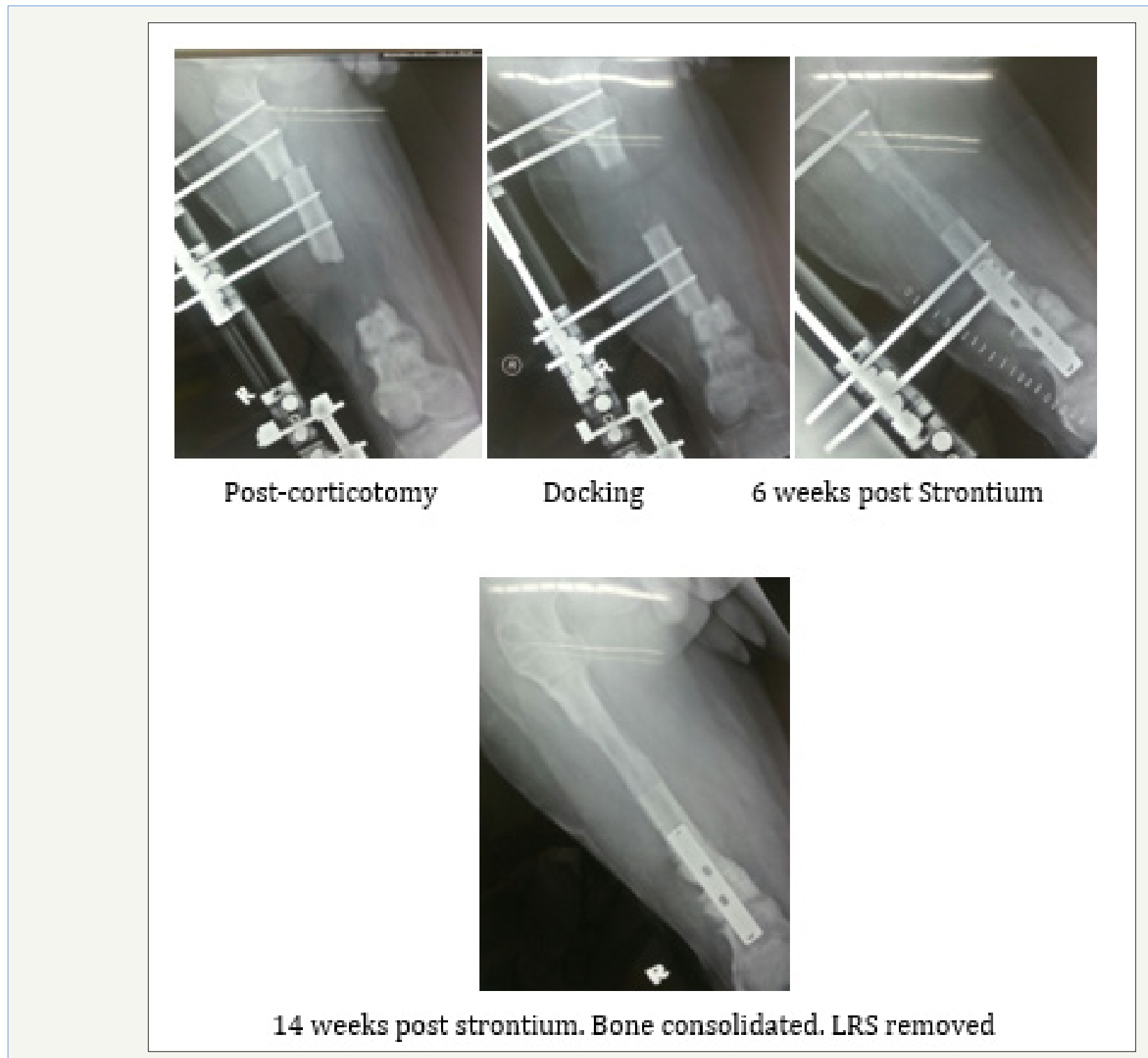

Figure 1:

Total length transported $=8.5 \mathrm{~cm}$

Total duration of transport $(0.5 \mathrm{~mm} \mathrm{BD})=87$ days

Expected duration of consolidation phase $=87 \times 2=174$ days

Expected total duration patient on LRS (transport+consolidation) $=261$ days

Total duration of consolidation phase after strontium $=98$ days

Total $=185$ days (Save 76 days).

\section{Patient 2}

17 years old boy sustained open fracture grade $3 \mathrm{~A}$ of right femur. However, patient opted for traditional treatment and came back 1 year later with malunited femur with $7 \mathrm{~cm}$ gross shortening and pus discharging sinus over the medial aspect of thigh. Working diagnosis was malunited femur with gross shortening and chronic osteomyelitis. Routine treatment was done, followed by gradual correction of deformity (rotation) and lengthening by Taylors
Spatial Frame. Total duration of correction+lengthening was 72 days. After achieving the desired length, patient was started on strontium $2 \mathrm{~g}$ OD to enhance bone consolidation. Below are the $\mathrm{x}$-rays of the patient.

In both our cases, the patients were started on strontium ranelate $2 \mathrm{~g} /$ day at the end of distraction phase and were continued throughout the consolidation phase until clinical union was achieved. The quality of regenerate bone on radiographs was 
normal. The end point for removal of external fixator was based on clinical union and radiological consolidation which was assessed by our senior orthopedic consultant. Both the patients were followed up for a minimum of 1 year and no complications were noted during the follow up period (Figure 2).

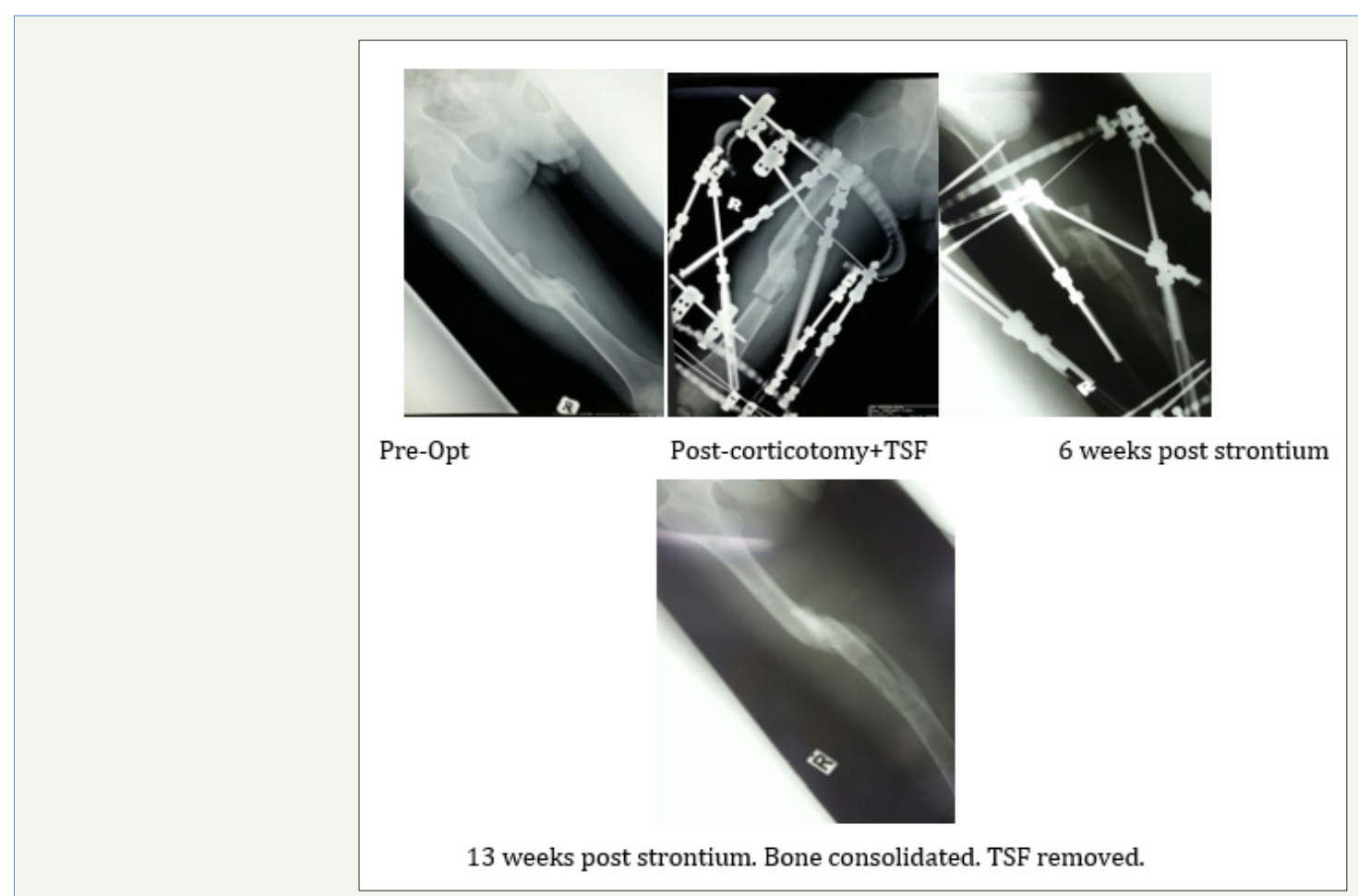

Figure 2:

Total length corrected $=7.0 \mathrm{~cm}$

Total duration of lengthening $=72$ days

Expected duration of consolidation phase $=72 \times 2=144$ days

Expected total duration patient on TSF (lengthening+ consolidation) $=216$ days

Total duration of consolidation after strontium $=91$ days

Total $=163$ (Save 53 days).

\section{Discussion}

Distraction osteogenesis is a surgical technique by which bone regeneration is induced between surgically osteotomized bone segments that undergo gradual distraction resulting in bone lengthening [4]. Codivilla was the first to explore distraction osteogenesis, and in 1904 reported applying this technique for lengthening of the lower limbs [1]. However, it was not until 1950 that this technique widespread through the works of Ilizarov. The common indications for distraction osteogenesis are open fractures, bone resection due to chronic osteomyelitis or infected non-union, large bone loss at initial time of trauma, limb length discrepancies and bony deformities $[1-7,16]$. It is often a limb salvage procedure but also has a role in cosmetic lengthening in short statures [2,9]. In distraction osteogenesis, bone heals by primary bone healing [17]. It results in new bone formation through the lengthening of the bone callus (also known ascallotasis). Induced traction generates tension within the callus and stimulates the formation of new bone parallel to the distraction vector. This force also creates tension on the surrounding soft tissues, initiating a sequence of adaptive changes known as distraction histogenesis [3]. Up to $10 \%$ lengthening is well-tolerated by muscle, but substantial histopathological changes occur after lengthening of $30 \%$ [1]. The gold standard for bone defect repairs is autograft but their usage is affected by morbidity at the donor site and limited supply. Limitations of the allograft include the immunogenic response by the host to the graft and the potential for disease transmission. Distraction osteogenesis can eliminate the need for bone grafts. Other known methods to treat large bone deficiencies are allografts, synthetic bone grafts, membrane induction technique or also known as Masquelet technique, cortical strut grafting, or vascularised bone grafting $[2-6,8,9]$. Some literatures also have mentioned acute shortening followed by bone lengthening later [7].

In distraction osteogenesis, there are three distinct phases: latent phase, distraction phase and consolidation phase. The latent phase is the interval between corticotomy and distraction and is usually 7-10 days [9]. However there are some literatures reported shorter latent phase in children and young adults [8]. Distraction phase is the interval between onset of distraction to the time of docking or end of transport. The normal distraction rate is $0.25 \mathrm{~mm}$ four times a day $(1 \mathrm{~mm} /$ day $)[2,3,9]$. During distraction, regenerate bone arises between the entire cross-sections of each distracted bone surface with a central radiolucent fibrous 
interzone comprising of type I collagen. New bone trabeculae form directly from this central collagen zone extending to both bone surfaces. However, the rate can vary depending on the quality of the regenerate bone. Consolidation phase is the end of distraction to bony unionor consolidation of the regenerate bone. It can be of 3 qualities: normal, hypertrophic of hypotrophic [8]. Up until now, the decision to discontinue distraction has been based more on personal experience than on objective data. None of the radiologic method used in assessing consolidation phase has given encouraging qualitative or quantitative results. However, a clinical study using scintography Tc99 DP has shown good results in determining end of consolidation phase.

Consolidation was considered to be reached when no more further changes in tracer uptake at the regenerate bone compared with surrounding normal growing bone [18]. As a general rule, the duration of consolidation phase is usually twice the duration of distraction phase [10]. One of the limitations of distraction osteogensesis is the long period of time required for the newly formed bone tissue to mineralize and consolidate. For example, if the bone deficiency is $10 \mathrm{~cm}$, the duration of distraction phase is 100 days $(0.25 \mathrm{mmx} 4$ /day) and the duration of consolidation is 200 days, which is a total of 300 days. During this whole period, patient has to be on an external fixator (Ilizarov rings or LRS). This period is known as 'frame time' [5]. Prolonged external fixation is uncomfortable for the patient and is associated with many complications such as pin site infections, hardware failure, mal-union, soft tissue contractures and poor cosmetic appearance $[2-4,6,9,16]$. Many methods have been introduced to decrease frame time; these include converting external fixator to internal fixator. Such examples are lengthening over a nail, lengthening with submuscular plating and lengthening by implantable device $[5,6]$. On the other hand, there are also methods been introduced to accelerate bone consolidation of the regenerated segment in bone transport, such as low intensity ultrasound therapy, BMP-2 injection, platelet rich plasma (PRP) injection, growth hormones, auto grafting and reaming through the new bone $[3,8,9]$. Unfortunately, these methods are invasive, not cost effective and are associated with high risk of deep infections.

Recently, usages of certain anti osteoporotic drugs have surfaced to promote bone healing. Many animal studies have demonstrated that these drugs can positively influence fracture repair and implant osseointegration $[12,15]$. Such examples of drugs are teraparatide (PTH), strontium ranelate, bisphosphonates and calcium supplements. In both our cases, we have used strontium ranelate. Strontium ranelate is a strontium slat in ranelic acid. Strontium nucleus is almost similar in size to that of calcium, the body easily absorbs it, incorporating it into the bones and tooth enamel instead of calcium It is a drug used in treatment of osteoporosis and it both increases deposition of new bone by osteoblasts and reduces the resorption of bone by osteoclasts $[11,1,2,14]$. Due to its both anabolic and anti-catabolic activity towards bone healing it is known as dual action bone agents (DABA) [11,1,2,14]. It acts through RANK/RANKL/OPG system [11]. In experimental studies in rats, it was found that strontium ranelate could promote fracture healing, increase callus volume, increase biomechanical strength and improving callus microstructural properties, without any negative effect on natural fracture healing progress [13]. In another animal study comparing strontium ranelate and PTH, it was found that both drugs increase callus volume but only strontium ranelae increases resistance to torsional testing [15]. The superior results obtained with strontium ranelate compared to PTH could be the consequence of a better quality of the new bone formed within the callus. In distraction osteogenesis, strontium ranelate stimulates production of osteoblasts (osteoinduction) and increases mineralisation of the regenerate segment. It promotes new bone formation, callus volume and bone healing, thus reduces the duration required for consolidation of the regenerate segment. Furthermore, it is also found to promote union at docking site, preventing a possibility of another surgery [13]. This not only reduces the total duration of patient being on external fixator (frame time), it also reduces the number of operations for the patient.

However, these observations are based only on our limited number of patients. Thus, further research and bigger clinical trial is required before we can safely conclude our hypothesis. Though most of the side effects of strontium ranelate are already elucidate, more analysis is required if strontium ranelate has to be used routinely in consolidating phase of bone transport.

\section{Conclusion}

Strontium ranelate is a cost effective and non-invasive alternative to reducing the duration of frame time in a patient undergoing distraction osteogenesis. Thus, it can be used to reduce patients' suffering, improve patients' compliance and reduce the disability period of a patient. However, further evaluation of the safety profile of the drug needs to be taken into consideration.

\section{References}

1. Spiegelberg B, Parratt T, Dheerendra SK, Khan WS, Jennings R (2010) Ilizarov principles of deformity correction. Ann R Coll Surg Engl 92(2): 101-105.

2. Dabkana TM, Nyaku FT, Bukar B (2016) Management of traumatic segmental bone loss using linear rail system, our experience at the university of maiduguri teaching hospital, maiduguri, Nigeria. Sahel Medical Journal 19(4): 171-174.

3. Knapp TR (1998) Distraction osteogenesis-poised for everyday use in orthopedic surgery. Surgery Distraction Osteogenesis 51(1905): 43-45.

4. Elsayed I, Abuomira A, Sala F, Elbatrawy Y, Lovisetti G, et al. (2016) Distraction osteogenesis for tibial nonunion with bone loss using combined ilizarov and taylor spatial frames versus a conventional circular frame. Strategies Trauma Limb Reconstr 11(3): 153-159.

5. Paley D, Harris M, Debiparshad K, Prince D (2014) Limb lengthening by implantable limb lengthening devices. Techniques in Orthopaedics 29(2): 72-85.

6. Li Z, Zhang X, Duan L, Chen X (2009) Distraction osteogenesis technique using an intramedullary nail and a monolateral external fixator in the reconstruction of massive postosteomyelitis skeletal defects of the femur. Can J Surg 52(2): 103-111.

7. Tetsworth AK, Sen C, Herzenberg JE, Jaffe M, Maar DC, et al. (2017) Bone transport versus acute shortening for the management of infected tibial non-unions with bone defects. Injury 48(10): 2276-2284.

8. Hamdy RC, Rendon JS, Tabrizian M (2011) Distraction osteogenesis and its challenges in bone regeneration. Bone Regeneration, pp. 178-204. 
9. Paley D (2014) Progress in and from Limb Lengthening 5: 1-27.

10. News LL (2011).

11. Zacchetti G, Dayer R, Rizzoli R, Ammann P (2014) Systemic treatment with strontium ranelate accelerates the filling of a bone defect and improves the material level properties of the healing bone. Biomed Res Int 2014: 549785.

12. Rafiq M, Ibrahim M, Singh S, Merican AM, Rao H, et al. (2016) The effect of strontium ranelate on the healing of a fractured ulna with bone gap in rabbit. BMC Vet Res 12(1): 1-9.

13. Tarantino U (2010) Strontium ranelate and bone healing: report of two cases 7(1): 65-68.

14. Fuleihan GE (2005) Strontium ranelate-a novel therapy for osteoporosis or a permutation of the same? The New England Journal of Medicine 350(5): 504-506
15. Bigi MM, Lewicki M, Ubios AM, Mandalunis PM (2011) Experimental model of distraction osteogenesis in edentulous rats. Braz Oral Res 25(3): 217-224.

16. Shyam AK, Singh SU, Modi HN, Song HR, Lee SH, et al. (2009) Leg lengthening by distraction osteogenesis using the Ilizarov apparatus : a novel concept of tibia callus subsidence and its influencing factors. Int Orthop 33(6): 1753-1759.

17. Middleton BR (2009) Molecular \& cellular aspects of bone growth in distraction osteogenesis. Nuffield Orthopaedic Centre 21.

18. Felemovicius J, Monasterio FO, Radillo LSG, Serna A (2000) Determining the optimal time for consolidation after distraction osteogenesis. J Craniofac Surg 11(5):430-436.

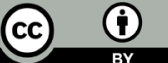

Creative Commons Attribution 4.0 International License

For possible submissions Click Here

\section{Submit Article}

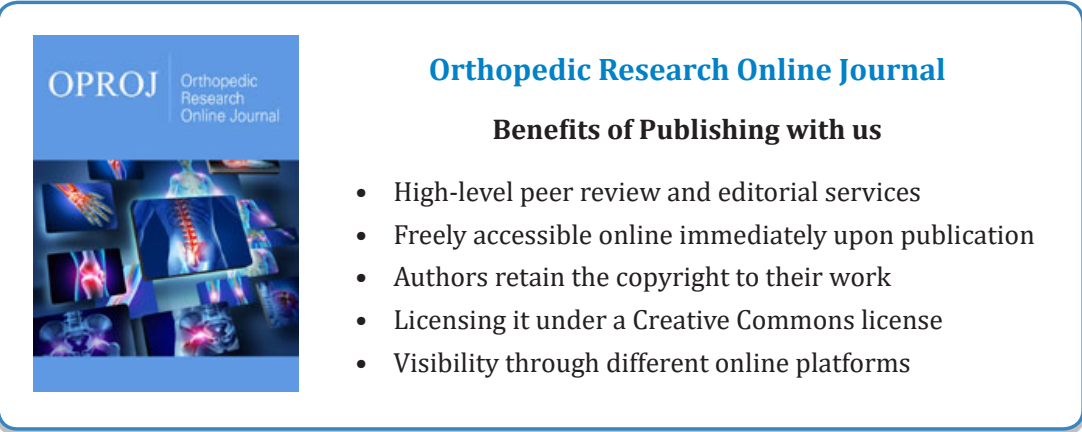

